\title{
Importance of multimodality imaging to guide therapy in stable CAD
}

\author{
Yuli Huang, Weiyi Mai and Yanxian Wu
}

We read the van der Hoeven Review (van der Hoeven, B. L. et al. Multimodality imaging in interventional cardiology. Nat. Rev. Cardiol. 9, 333-346 [2012]) ${ }^{1}$ with great interest. We wish to discuss the important role of multimodality imaging in the management of patients with stable coronary artery disease (CAD).

With the rising prevalence of $\mathrm{CAD}$ and the associated costs of health care, the optimal therapeutic strategy for patients with stable CAD has been discussed widely over the past decade. Most randomized clinical trials, such as the Clinical Outcomes Utilizing Revascularization and Aggressive Drug Evaluation (COURAGE) ${ }^{2}$ and the Bypass Angioplasty Revascularization Investigation 2 Diabetes (BARI 2D), ${ }^{3}$ failed to show a reduction in mortality or the incidence of myocardial infarction with revascularization compared with optimal medical therapy. A newly published metaanalysis supports this finding. ${ }^{4}$ However, in almost all these studies, randomization occurred after coronary angiography. Whether all patients need to undergo coronary angiography, and how to perform risk stratification by noninvasive testing remain unresolved.

Stress imaging with single-photon emission computed tomography (SPECT) provides substantial prognostic information in patients with stable CAD. ${ }^{5}$ A large, retrospective study involving $>10,000$ patients assessed with SPECT showed that the benefits of revascularization were confined to patients with $\geq 10 \%$ left ventricular ischemia. ${ }^{6}$ Subsequently, the same research group expanded the results by retrospectively analyzing $>13,000$ patients, and found that those with a threshold of $>10-15 \%$ ischemia without extensive scarring seem to benefit from revascularization. ${ }^{7}$ Similarly, Zellweger et al. reported that, in 3,664 patients with 'silent', asymptomatic ischemia, only those with $\geq 7.5 \%$ ischemia of the myocardium benefited from revascularization. ${ }^{8}$ Moreover, Sorajja et al. observed that, in asymptomatic patients with diabeties mellitus, surgical revascularization was beneficial only in patients determined by SPECT as being 'high risk.' These observational studies highlight the importance of the degree of ischemia in risk stratification and management of patients with stable CAD.

Subgroup analyses of COURAGE ${ }^{10}$ and BARI 2D ${ }^{11}$ also showed that SPECT provides important information regarding prognosis in patients with stable CAD. However, determination of therapeutic strategy for these patients on the basis of the degree of ischemia on stress imaging has not been prospectively tested in randomized trials. Investigators in the International Study of Comparative Health Effectiveness with Medical and Invasive Approaches (ISCHEMIA) are currently randomly allocating patients with documented ischemia ( $>10 \%$ of left ventricular mass) by noninvasive stress testing to optimal medical therapy or revascularization. Patients with left main CAD or nonobstructive CAD will be excluded by coronary CT assessment before randomization. ${ }^{5,12} \mathrm{We}$ await the results of this trial, which might provide helpful information to optimize the management of patients with stable CAD.

In their Review, ${ }^{1}$ van der Hoeven and colleagues discuss the combination of myocardial perfusion imaging and assessment of coronary artery anatomy by CT coronary angiography to refine the therapeutic strategy, reduce the risk of unnecessary invasive angiography, and maximize the effectiveness of revascularization. Co-registration of CT with SPECT or PET in clinical practice can allow comprehensive, noninvasive evaluation of CAD, including both morphological and functional assessment. However, about half of the patients who underwent selective percutaneous coronary intervention for stable angina in either the UK in 2007 or the USA in 2004 did not receive a stress test to assess ischaemia. ${ }^{13}$ Although no data on this issue are available in China to our knowledge, the situation is unlikely to be better than in the UK or USA. Given the high prevalence of CAD worldwide and the number of inappropriate revascularization procedures, we strongly advocate the comprehensive, noninvasive evaluation of CAD burden by multimodality imaging to determine the appropriate therapeutic strategy.

Department of Cardiology, the Affiliated Hospital at Shunde (the First People's Hospital of Shunde), Southern Medical University, Penglai Road 1, Daliang Town, Shunde District, Foshan 528,300, China (Y. Huang, Y. Wu). Department of Cardiology, the First Affiliated Hospital of Sun Yat-sen University, 58 Zhongshan Road 2, Guangzhou, 510,080, China (W. Mai).

Correspondence to: $Y . W u$ wuyanxian1064@163.com

\section{Acknowledgments}

The authors of this manuscript are funded by the Medical Scientific Research Grant of the Health Ministry of Guangdong province, China (No. B2011310, A2012663), and the Scientific Research Fund of Foshan, Guangdong, China (No. 201,108,204).

Competing interests

The authors declare no competing interests.

1. van der Hoeven, B. L., Schalij, M. J. $\&$ Delgado, V. Multimodality imaging in interventional cardiology. Nat. Rev. Cardiol. 9, 333-346 (2012).

2. Boden, W. E. et al. Optimal medical therapy with or without $\mathrm{PCl}$ for stable coronary disease. N. Engl. J. Med. 356, 1503-1516 (2007).

3. Frye, R. L. et al. A randomized trial of therapies for type 2 diabetes and coronary artery disease. N. Engl. J. Med. 360, 2503-2515 (2009).

4. Stergiopoulos, K. \& Brown, D. L. Initial coronary stent implantation with medical therapy vs medical therapy alone for stable coronary artery disease: meta-analysis of randomized controlled trials. Arch. Intern. Med. 172, 312-319 (2012).

5. Farzaneh-Far, A. \& Borges-Neto, S. Ischemic burden, treatment allocation, and outcomes in stable coronary artery disease. Circ. Cardiovasc. Imaging 4, 746-753 (2011). 
6. Hachamovitch, R., Hayes, S. W., Friedman, J. D., Cohen, I. \& Berman, D. S. Comparison of the short-term survival benefit associated with revascularization compared with medical therapy in patients with no prior coronary artery disease undergoing stress myocardial perfusion single photon emission computed tomography. Circulation 107, 2900-2907 (2003).

7. Hachamovitch, R. et al. Impact of ischaemia and scar on the therapeutic benefit derived from myocardial revascularization vs. medical therapy among patients undergoing stress-rest myocardial perfusion scintigraphy. Eur. Heart J. 32, 1012-1024 (2011).

8. Zellweger, M. J. et al. Threshold, incidence, and predictors of prognostically high-risk silent ischemia in asymptomatic patients without prior diagnosis of coronary artery disease. J. Nucl. Cardiol. 16, 193-200 (2009).

9. Sorajja, P. et al. Improved survival in asymptomatic diabetic patients with high-risk SPECT imaging treated with coronary artery bypass grafting. Circulation 112 (Suppl. 9), |311-|316 (2005).

10. Shaw, L. J. et al. Optimal medical therapy with or without percutaneous coronary intervention to reduce ischemic burden: results from the Clinical Outcomes Utilizing Revascularization and Aggressive Drug Evaluation (COURAGE) trial nuclear substudy. Circulation 117, 1283-1291 (2008).

11. Shaw, L. J. et al. Impact of left ventricular function and the extent of ischemia and scar by stress myocardial perfusion imaging on prognosis and therapeutic risk reduction in diabetic patients with coronary artery disease: results from the Bypass Angioplasty Revascularization Investigation 2 Diabetes (BARI 2D) trial. J. Nucl. Cardiol. http:// dx.doi.org/10.1007/s12350-012-9548-3.

12. Songco, A. V. \& Brener, S. J. Initial strategy of revascularization versus optimal medical therapy for improving outcomes in ischemic heart disease: a review of the literature. Curr. Cardiol. Rep. http://dx.doi.org/10.1007/ s11886-012-0278-6.

13. Simoons, M. L. \& Windecker, S. Chronic stable coronary artery disease: drugs vs. revascularization. Eur. Heart J. 31, 530-541 (2010). 